\title{
Transitional Flow in Tubes: Experimental Results and Recommended Correlations for Calculation of Pressure Drop and Heat Transfer in Plain and Micro-fin Tubes
}

\author{
Afshin J. Ghajar \\ Regents Professor and John Brammer Endowed Professor School of Mechanical and Aerospace Engineering \\ Oklahoma State University \\ Stillwater, Oklahoma, USA \\ afshin.ghajar@okstate.edu
}

For proper design of thermal systems such as compact heat exchangers involving horizontal tubes that are heated by a uniform wall heat flux boundary condition, proper knowledge of the friction factor (pressure drop) and heat transfer coefficient in the tube for the entrance and fully developed regions with different inlet geometries under different flow regimes (laminar, transitional, and turbulent) is essential. In practical engineering design, the usual recommendation is to avoid design and operation in this region, but this is not always feasible under design constraints. The usually cited transitional Reynolds number range of about 2300 (onset of turbulence) to 10,000 (fully turbulent condition) applies, strictly speaking, to a very steady and uniform entry flow with a rounded entrance. If the flow has a disturbed entrance typical of heat exchangers in which there is a sudden contraction and possibly even a re-entrant entrance, the transitional Reynolds number range will be very different.

In a series of studies, Ghajar and coworkers experimentally investigated the inlet configuration effects on the developing and fully developed transitional pressure drop (friction factor) under isothermal and heating (uniform wall heat flux boundary) conditions and developing and fully developed transitional forced and mixed convection heat transfer in plain and micro-fin circular horizontal tubes. On the basis of their experimental data, they developed practical and easy to use correlations for the friction factor (pressure drop) and heat transfer coefficient in the transition region as well as the laminar and turbulent flow regions.

This lecture provides a detailed discussion of transitional flows in tubes, with particular emphasis on the entrance geometry and the development region. The discussion also deals with flows that may exhibit buoyant motion and property variations. 